09

\title{
Решетки населенностей, создаваемые в газе атомов водорода с помощью ультрафиолетовых аттосекундных импульсов
}

\author{
(C) Р.М. Архипов ${ }^{1,2}$, М.В. Архипов ${ }^{1}$, А.В. Пахомов ${ }^{1}$, Ю.М. Артемьев ${ }^{1}$, Н.Н. Розанов ${ }^{2}$ \\ ${ }^{1}$ Санкт-Петербургский государственный университет, \\ 199034 Санкт-Петербург, Россия \\ ${ }^{2}$ ФТИ им. А.Ф. Иофффе, \\ 194021 Санкт-Петербург, Россия \\ e-mail: arkhipovrostislav@gmail.com, m.arkhipov@spbu.ru, antpakhom@gmail.com, nnrosanov@mail.ru
}

Поступила в редакцию 23.01.2021 г.

В окончательной редакции 23.01.2021 г.

Принята к публикации 04.02.2021 г.

Исследована возможность создания решеток населенностей в газе атомов водорода с помощью пары ультрафиолетовых (УФ, UV) аттосекундных импульсов, которые не перекрываются в среде. При этом центральная частота импульсов может как совпадать с частотой резонансного перехода $1-2$ из основного состояния в первое возбужденное (основная линия серии Лаймана), так и быть отстроенной от него. Результаты численных расчетов согласуются с аналитическими значениями, полученными на основании приближенного решения уравнения Шредингера по теории возмущений. Показано, что при резонансном возбуждении наибольшая эффективность создания решеток достигается с увеличением длительности импульсов. При нерезонансном возбуждении, напротив, система эффективнее возбуждается короткими квазиуниполярными субцикловыми импульсами, чем биполярными многоцикловыми. Полученные результаты могут быть применимы к когерентному возбуждению единичного атома (тонкого слоя) с помощью пары UV-импульсов. Показана возможность управления глубиной модуляции решеток с помощью изменения фазы (carrier envelope phase, CЕР) аттосекундных импульсов.

Ключевые слова: аттосекундные импульсы, светоиндуцированные решетки, атом водорода.

DOI: $10.21883 /$ OS.2021.05.50890.1842-21

\section{Введение}

Генерация аттосекундных импульсов в ультрафиолетовом (UV) и оптическом диапазонах стала одной из центральных тем в современной физике за последние десятилетия [1-11]. Период оборота электрона по первой классической боровской орбите в атоме водорода порядка 150 as. Поэтому получение таких импульсов позволяет выполнять фундаментальные исследования по изучению и управлению движением электронов в атомaх, молекулах, твердых телах и наноструктурах [6,7]. Обычно получаемые на практике аттосекундные и фемтоскундные импульсы являются биполярными - в них вектор напряженности электрического поля за период импульса много раз меняет знак, и электрическая площадь (интеграл от напряженности электрического поля по времени $S_{E}=\int E(t) d t$ ) равна 0 . При дальнейшем сокращении длительности импульсов до одной полуволны поля удается получить уже униполярные полупериодные импульсы [8-10]. Их площадь уже может быть отлична от 0. Подчеркнем, что характер воздействия таких униполярных импульсов на атомные системы отличается от воздействия обычных многоцикловых биполярных. Ввиду однонаправленного воздействия на электроны такие импульсы эффективнее воздействуют на квантовые системы по сравнению с биполярными импульсами [12], что позволяет селективно возбуждать те или иные пере- ходы в атомах [13,14], ускорять заряженные частицы [15] и управлять их спином $[16,17]$. Возможные применения униполярного субциклового света резюмированы в об3оре [10].

Типичные времена релаксации населенностей $T_{1}$ и поляризации $T_{2}$ в газовых средах лежат в наносекундном диапазоне, что существенно длиннее импульсов возбуждения, длительность которых лежит в фемто-(атто-) секундном диапазоне. Поэтому взаимодействие таких импульсов со средой является когерентным [18]. При когерентном взаимодействии малоциклового импульса co средой за счет осцилляций Раби (carrier wave Rabi flopping) [19] они способны сравнительно быстро изменять населенности среды за время порядка длительности импульсов. Так, если в среде навстречу друг другу распространяется пара коротких импульсов, не встречающихся в среде, и задержки между ними меньше времени когерентности среды $T_{2}$, то это может привести к созданию в среде периодически изменяющегося распределения населенностей атомных уровней - решетки населенностей [20-23]. О возможности их создания в оптике сообщалось еще в первых экспериментальных работах по фотонному эху, в которых использовались длинные импульсы наносекундной длительности [20-22]. Таким образом, при когерентном взаимодействии для наведения решеток не требуется перекрытие падающих пучков в среде в отличие от стандарного подхода, в 


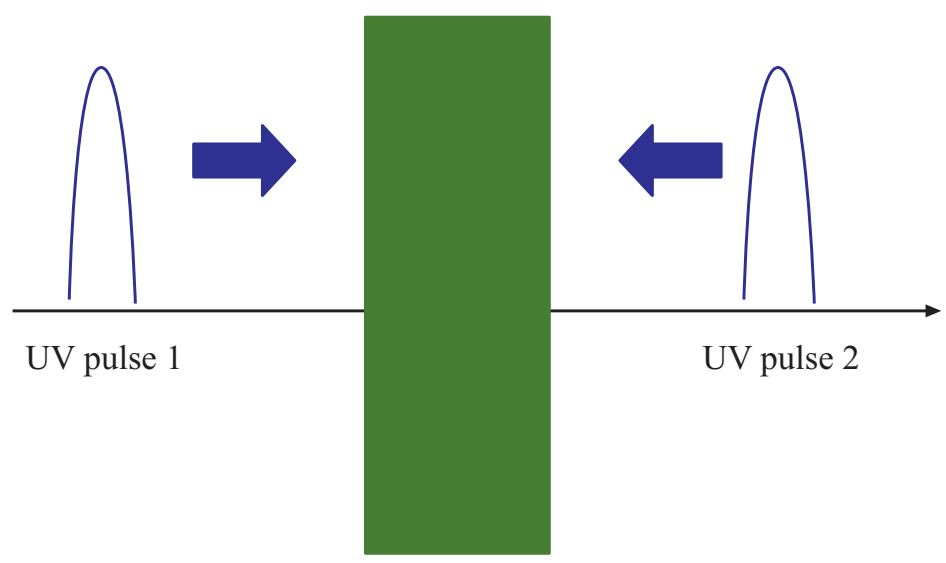

$a$

Gas of hydrogen atoms
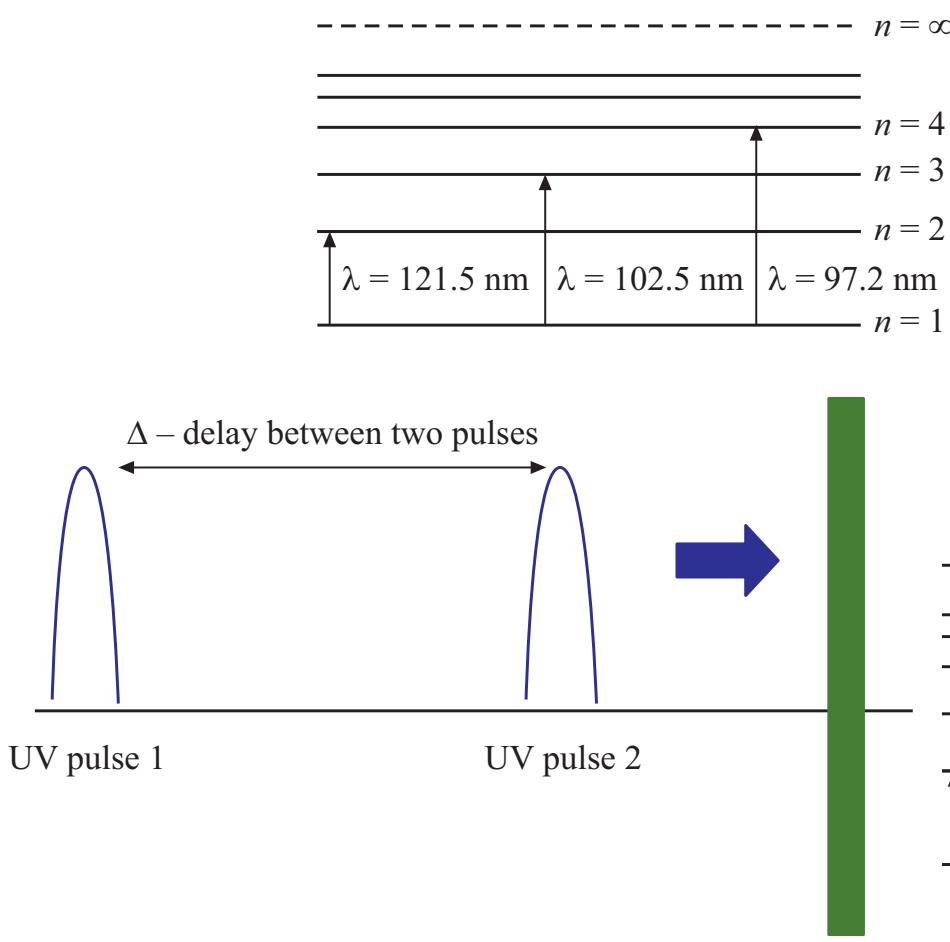

Thin layer of the atomic medium (e.g. hydrogen atoms)

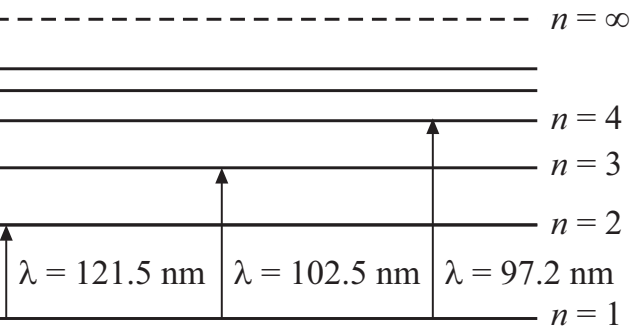

Рис. 1. (a) Возбуждение протяженного газа атомов водорода парой UV аттосекундных импульсов, распространяющихся навстречу друг другу и не встречающихся в среде, $(b)$ когерентное возбуждение тонкого слоя атомной среды (водорода) парой импульсов, распространяющихся с некоторой задержкой друг относительно друга.

котором решетки создаются при интерференции двух перекрывающихся в среде квазимонохроматических световых пучков [24]. В работе [23] была показана возможность измерения времени релаксации поляризации $T_{2}$ в резонансной среде с помощью наведенной таким образом решетки.

Но возможность создания решеток с помощью не перекрывающихся в среде пучков после упомянутых работ так и не нашла значительных применений в оптике и фактически до настоящего времени была незаслуженно забыта. Интерес к этой теме возник только в последние годы после появления предельно коротких фемтосекундных и аттосекундных импульсов [25-29]. Данная возможность создания решеток изучалась на- ми в двухуровневой среде, когда решетки наводились последовательностью аттосекундных импульсов в оптическом диапазоне [25,26]. Впоследствии она была изучена в трехуровневых и многоуровневых системах на примере паров рубидия, возбуждаемого униполярными импульсами [27,28]. Из этих исследований следует, что для получения решеток с помощью аттосекундных импульсов в оптическом диапазоне в атомах требуются очень большие значения амплитуды напряженностей поля ( $\left.10^{6} \mathrm{~V} / \mathrm{cm}\right)$, что трудно получить на практике. Для того чтобы снизить напряженность поля $\left(\sim 10^{3} \mathrm{~V} / \mathrm{cm}\right)$, в [29] было предложено наводить решетки в ТГц диапазоне частот на колебательных переходах в молекулах с большими дипольными моментами пере- 
ходов с помощью последовательности ТГц импульсов, которые легче получить экспериментально [30]. Также в [29] на основании приближенного решения уравнения Шредингера в приближении слабого поля была развита теория создания и управления решетками населенностей в многоуровневых средах. Полученные в [29] выражения являются универсальными, так как получены на основании решения уравнения Шредингера.

В настоящей работе на основании разработанной в [29] общей теории изучается возможность создания решеток населенностей в простейшей квантовой системе - протяженной среде газа атомов водорода (рис. $1, a)$, а также когерентное возбуждение единичного атома водорода (или тонкого слоя атомов водорода, рис. $1, b)$ UV аттосекундными импульсами. При этом рассматривается случай как резонансного, так и нерезонансного возбуждения. Показана возможность эффективного создания решеток и эффективного возбуждения одиночного атома с помощью униполярных полуцикловых импульсов при нерезонансном взаимодействии. Изучается возможность контроля глубины модуляции решеток (возбуждения) при управлении фазой (carrier envelope phase, CEP) аттосекундных импульсов.

\section{Создание решеток в протяженном газе атомов водорода при резонансном возбуждении парой UV аттосекундных импульсов}

Пусть на протяженную среду (газ) атомов водорода воздействует пара UV аттосекундных импульсов, распространяющихся навстречу друг другу и не встречающихся в среде (рис. 1,a):

$$
\begin{aligned}
E_{a}(t) & =E_{0 a} e^{-\frac{t^{2}}{\tau^{2}}} \cos (\Omega t+\phi) \\
& +E_{0 a} e^{\frac{-(t-\Delta)^{2}}{\tau^{2}}} \cos (\Omega[t-\Delta]+\phi) .
\end{aligned}
$$

Здесь $\Omega$ - центральная частота аттосекундных импульсов, $\phi$ - фаза (carrier envelope phase, CEP), $\Delta-$ задержка между импульсами. В случае тонкого слоя среды с малой плотностью частиц изменением формы импульсов при распространении можно пренебречь. Поэтому их воздействие на атомы среды, локализованные в некоторой точке пространства, можно описывать в терминах задержки $\Delta$, которая определяет момент прихода 2-го импульса в точку среды с координатой $z$ [25-29]. Схема энергетических уровней атома водорода схематически показана на рис. 1. Величина энергии зависит от главного квантового числа $n$, и расстояние между уровнями спадает с ростом $n$ [31].

Пусть система до прихода импульсов находилась в основном состоянии с $n=1$. В [29] в первом порядке теории возмущений было получено следующее выражение для населенности $n$-го состояний системы после
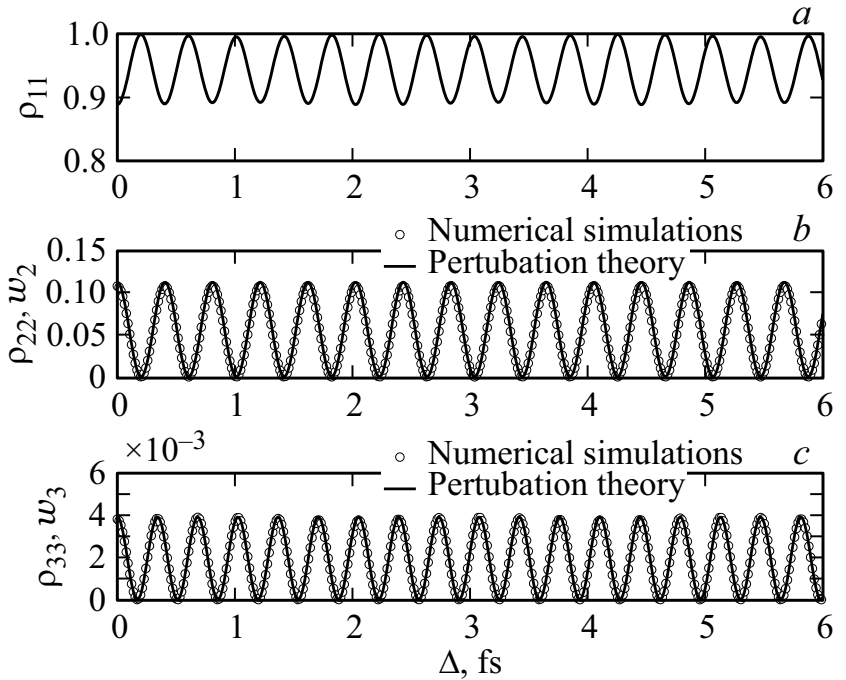

Рис. 2. Зависимости населенности уровней $\rho_{11}, \rho_{22}, \rho_{33}$ от задержки между аттосекундными импульсами $\Delta$. Сплошная линия - результат аналитического расчета по формуле (2), кружками показан результат численного расчета с помощью системы уравнений (3)-(9). Параметры расчета указаны в табл. 1.

импульсов:

$$
\begin{aligned}
w_{n} & =\frac{d_{1 n}^{2}}{\hbar^{2}} E_{0}^{2} \tau^{2} \exp \left[-\frac{\left(\omega_{1 n}^{2}+\Omega^{2}\right)}{2}\right] \\
& \times\left[\cosh \left(\omega_{1 n} \Omega \tau^{2}\right)+\cos 2 \phi\right]\left[1+\cos \left(\omega_{1 n} \Delta\right)\right] .
\end{aligned}
$$

Здесь $d_{1 n}$ и $\omega_{1 n}$ - дипольный момент и частота перехода. Из (2) видна периодическая зависимость населенностей уровней от задержки между импульсами, что говорит о возможности создания гармонической решетки населенностей в протяженной среде, глубина модуляции которой зависит от фазы импульса $\phi$ и его частоты $\Omega$. А в случае единичного атома (рис. $1, b$ ) формула (2) иллюстрируют возможность селективного возбудения/девозбуждения резонансных переходов среды с помощью пары предельно коротких импульсов при изменения задержки между ними $[13,14]$.

Для изучения возможности создания решеток населенностей мы рассчитывали их аналитически по формуле (2) и с помощью численного решения системы уравнений для матрицы плотностей трехуровневой среды, параметры в которой соответствуют переходам в атоме водорода. Она имеет вид [32]

$$
\begin{aligned}
\frac{\partial}{\partial t} \rho_{21} & =-i \omega_{21} \rho_{21}-i \frac{d_{12}}{\hbar} E\left(\rho_{22}-\rho_{11}\right) \\
& -i \frac{d_{13}}{\hbar} E \rho_{23}+i \frac{d_{23}}{\hbar} E \rho_{31}, \\
\frac{\partial}{\partial t} \rho_{32} & =-i \omega_{32} \rho_{32}-i \frac{d_{23}}{\hbar} E\left(\rho_{33}-\rho_{22}\right) \\
& -i \frac{d_{12}}{\hbar} E \rho_{31}+i \frac{d_{13}}{\hbar} E \rho_{21},
\end{aligned}
$$


Таблица 1. Параметры модели и возбуждающих импульсов

\begin{tabular}{|c|c|}
\hline $\begin{array}{l}\text { Частота перехода } 12 \text { в атоме водорода } \\
\text { (длина волны перехода) }\end{array}$ & $\omega_{21}=1.55 \cdot 10^{16} \mathrm{rad} / \mathrm{s}\left(\lambda_{21}=121.6 \mathrm{~nm}\right)$ \\
\hline Дипольный момент перехода 12 & $d_{12}=3.27 \mathrm{D}$ \\
\hline $\begin{array}{l}\text { Частота перехода } 13 \text { в атоме водорода } \\
\text { (длина волны перехода) }\end{array}$ & $\begin{array}{c}\omega_{31}=1.837 \cdot 10^{16} \mathrm{rad} / \mathrm{s} \\
\left(\lambda_{31}=102.6 \mathrm{~nm}\right)\end{array}$ \\
\hline Дипольный момент перехода 13 & $d_{13}=1.31 \mathrm{D}$ \\
\hline $\begin{array}{l}\text { Частота перехода } 23 \text { в атоме водорода } \\
\text { (длина волны перехода) }\end{array}$ & $\begin{array}{c}\omega_{32}=2.87 \cdot 10^{15} \mathrm{rad} / \mathrm{s} \\
\left(\lambda_{32}=656.6 \mathrm{~nm}\right)\end{array}$ \\
\hline Дипольный момент перехода 23 & $d_{23}=7.78 \mathrm{D}$ \\
\hline Амплитуда аттосекундных импульсов возбуждения & $E_{0 a}=3 \cdot 10^{7} \mathrm{~V} / \mathrm{cm}$ \\
\hline $\begin{array}{l}\text { Центральная частота (длина волны) } \\
\text { возбуждения }\end{array}$ & $\begin{array}{c}\Omega=\omega_{21}=1.55 \cdot 10^{16} \mathrm{rad} / \mathrm{s} \\
\text { (длина волны } \lambda_{a}=2 \pi / \Omega=121.6 \mathrm{~nm} \text { ) }\end{array}$ \\
\hline $\begin{array}{l}\text { Длительность аттосекундных } \\
\text { импульсов возбуждения }\end{array}$ & $\tau=608$ as \\
\hline Фаза (CЕР) & $\phi=0$ \\
\hline
\end{tabular}

$$
\begin{gathered}
\frac{\partial}{\partial t} \rho_{31}=-i \omega_{31} \rho_{31}-i \frac{d_{13}}{\hbar} E\left(\rho_{33}-\rho_{11}\right) \\
-i \frac{d_{12}}{\hbar} E \rho_{32}+i \frac{d_{23}}{\hbar} E \rho_{21}, \\
\frac{\partial}{\partial t} \rho_{11}=i \frac{d_{12}}{\hbar} E\left(\rho_{21}-\rho_{21}^{*}\right)-i \frac{d_{13}}{\hbar} E\left(\rho_{13}-\rho_{13}^{*}\right), \\
\frac{\partial}{\partial t} \rho_{22}=i \frac{d_{12}}{\hbar} E\left(\rho_{21}-\rho_{21}^{*}\right)-i \frac{d_{23}}{\hbar} E\left(\rho_{23}-\rho_{23}^{*}\right), \\
\frac{\partial}{\partial t} \rho_{33}=i \frac{d_{13}}{\hbar} E\left(\rho_{13}-\rho_{13}^{*}\right)+i \frac{d_{23}}{\hbar} E\left(\rho_{23}-\rho_{23}^{*}\right) .
\end{gathered}
$$

Здесь $\rho_{21}, \rho_{32}, \rho_{31}$ - недиагональные элементы матрицы плотности, определяющие динамику поляризации среды, $\rho_{11}, \rho_{22}, \rho_{33}$ - населенности 1-го, 2-го и 3-го состояний атома соответственно, $d_{12}, d_{13}, d_{23}$ - дипольные моменты переходов, $\omega_{21}, \omega_{32}, \omega_{31}$ - частоты переходов, $\hbar$ - приведенная постоянная Планка. Релаксационными членами мы пренебрегли из-за малой длительности импульсов возбуждения.

Параметры модели (частоты переходов и дипольные моменты переходов) были взяты, как в атоме водорода [31], табл. 1. Центральная частота возбуждающих импульсов $\Omega=\omega_{21}$ совпадает с частотой резонансного перехода из основного в первое возбужденое состояние 1-2 серии Лаймана в атоме водорода с длиной волны $121.6 \mathrm{~nm}$.

Зависимость населенностей уровней после окончания действия импульсов, рассчитанная по формуле (2) и с помощью численного решения системы уравнений (3)-(8), показана на рис. 2 сплошной линии и кружками соответственно. Параметры расчета приведены в табл. 1. Видно, что результаты, полученные по формуле (2), хорошо согласуются с численными расчетами. Аналогичное согласие наблюдалось при нерезонансном

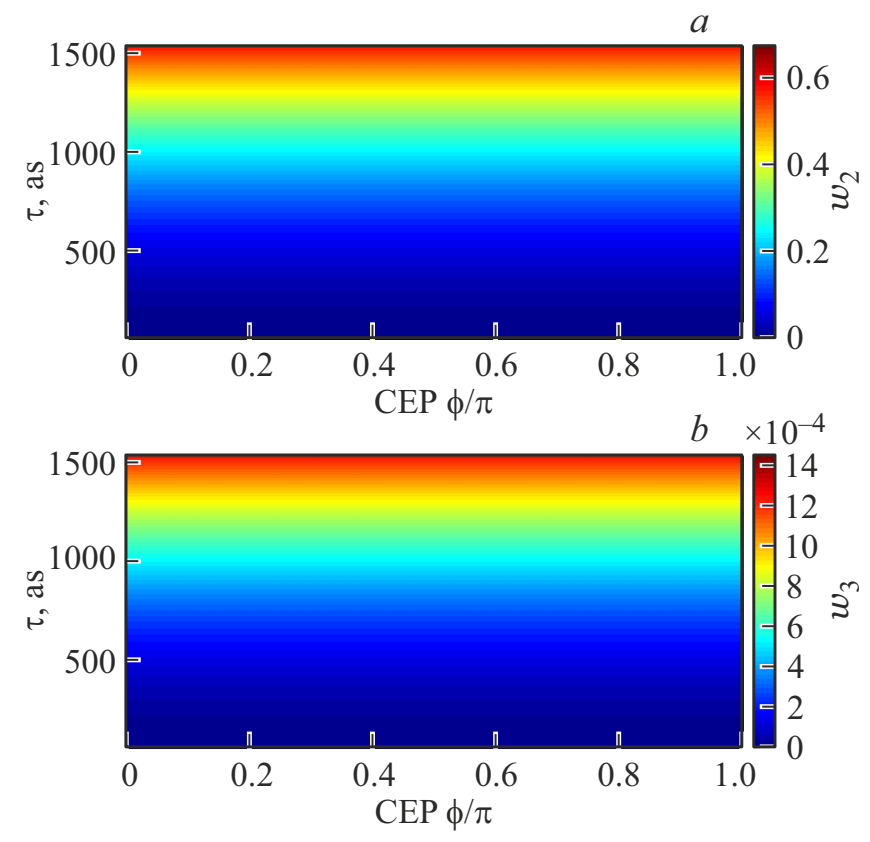

Рис. 3. (a) Зависимость населенностей второго $w_{2}$ и третьего $w_{3}(b)$ уровней в газе атомов водорода от длительности возбуждающих импульсов $\tau$ и фазы СЕР $\phi$ при величине задержки $\Delta=6$ fs. Остальные параметры указаны в табл. 1 .

возбуждении среды униполярными импульсами без несущей частоты $(\Omega=0)$ в $[28,29]$. Но в данном случае среда возбуждается резонансными биполярными импульсами с несущей частотой, равной частоте перехода 1-2 основной линии серии Лаймана в водороде, $\Omega=\omega_{21}$.

Также из рис. 2 и табл. 1 видно, что для создания решеток требуются большие значения напряженности 
Таблица 2. Параметры импульсов возбуждения

\begin{tabular}{l|c}
\hline $\begin{array}{l}\text { Амплитуда UV аттосекундных } \\
\text { импульсов возбуждения }\end{array}$ & $E_{0 a}=3 \cdot 10^{7} \mathrm{~V} / \mathrm{cm}$ \\
\hline $\begin{array}{l}\text { Центральная частота } \\
\text { (длина волны) возбуждения }\end{array}$ & $\begin{array}{c}\Omega=\frac{\omega_{21}}{2}=7.75 \cdot 10^{15} \mathrm{rad} / \mathrm{s} \\
\left(\text { длина волны } \lambda_{a}=243.2 \mathrm{~nm}\right)\end{array}$ \\
\hline Фаза (CEP) & $\phi=0$
\end{tabular}

поля $E_{0 a}=3 \cdot 10^{7} \mathrm{~V} / \mathrm{cm}$, как и в двухуровневой среде $[25,26]$. Из формулы (2) также видно, что управлять глубиной модуляции решеток в атомах можно с помощью изменения фазы СЕР аттосекундных импульсов. Рисунок 3 иллюстрирует населенности уровней 2 и 3 в зависимости от длительности импульсов возбуждения и фазы CEP.

Из него следует практическое отсутствие зависимости населенностей $w_{2}$ и $w_{3}$ от фазы $\phi$ и рост $w_{2}$ при увеличении длительности импульса $\tau$ (рис. 3, $a$ ). Это довольно очевидный результат - при резонансном возбуждении, когда $\omega_{12}=\Omega$, чем больше длительность импульса, тем отчетливее проявляется его воздействие и тем больше населенность уровня 2 (рис. 3,a). А населенность 3-го уровня $w_{3}$ значительно ниже (рис. $3, b)$, так как частота перехода 1-3 отстроена от частоты импульса $\Omega$. Поэтому для создания решеток co значительной глубиной модуляции при резонансном возбуждении нужно использовать длинные импульсы и большие значения амплитуды полей возбуждения.

Значительное влияние фазы СЕР на глубину модуляции решеток проявляется при переходе к более коротким длительностям возбуждающих импульсов субцикловым и униполярным полупериодным, когда взаимодействие со средой уже нерезонансное. Данная ситуация будет рассмотрена в следующем разделе.

\section{Решетки населенности \\ в протяженной среде атомов водорода при нерезонансном возбуждении парой UV аттосекундных импульсов}

Теперь рассмотрим возможность создания решеток в протяженном газе атомов водорода при нерезонансном воздействии пары квазиуниполярных UV аттосекундных импульсов. Параметры возбуждения приведены в табл. 2.

Рисунок 4 иллюстрирует зависимость населенности второго и третьего состояний $w_{2}$ и $w_{3}$ в водороде от длительности возбуждающих импульсов и задержки между ними, рассчитанную по формуле (2).

Видно, что в отличие от резонансного взаимодействия (рис. 3) для достижения большой глубины модуляции необходимо использовать более короткие импульсы с

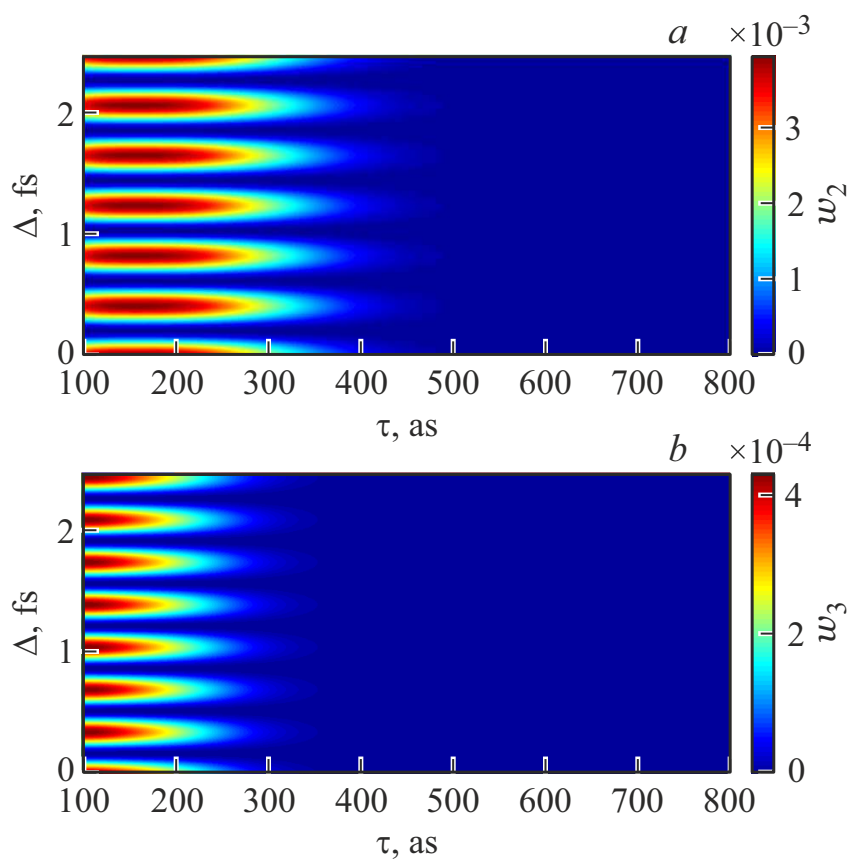

Рис. 4. (a) Зависимость $w_{2}$ и $w_{3}(b)$ в газе атомов водорода от длительности возбуждающих импульсов $\tau$ и задержки $\Delta$. Остальные параметры указаны в табл. 2.

длительностью в интервале 100-200 as, равной доле периода $T_{p}=\frac{2 \pi}{\Omega}=810$ as. При этом длинные многоцикловые импульсы с длительностью порядка $T_{p}$ и выше не могут создать решетку со значительной глубиной модуляции. При длительностях короче периода $T_{p}$ возбуждающие импульсы являются квазиуниполярными. Это значит, что квазиуниполярные полупериодные импульсы способны оказывать более эффективное воздействие на атом, чем моногоцикловые биполярные, что согласуется с ранее сделанными выводами [12-14,29].

Рисунок 5 иллюстрирует зависимость $w_{2}$ и $w_{3}$ от фазы $\phi$ и длительности импульсов $\tau$. Видно, что теперь населенности сильно зависят от $\phi$. Они принимают наибольшие значения, когда абсолютная величина электрической площади импульca $S_{E}=E_{0} \tau \sqrt{\pi} \exp \left(-\Omega^{2} \tau^{2} / 4\right) \cos \phi$ максимальна (при $\phi=0, \pi)$ и длительность импульса мала $\Omega \tau \ll 1$, т.е. в случае квазиуниполярных импульсов. Длинные импульсы, для которых $\Omega \tau \gg 1$, практически не возбуждают среду. Аналогичные результаты были получены в ТГц диапазоне частот при возбуждении молекулярных сред [29].

Зависимости населенностей от длительности $\tau$ и амплитуды $E_{0}$ возбуждающих импульсов, также рассчитанные по формуле (2), показана на рис. 6. При этом для достижения большой глубины модуляции решеток, как видно из этого рисунка, необходимо также использовать короткие импульсы и увеличивать их амплитуду. 


\section{Управление населенностями состояний единичного атома водорода (тонкого слоя) с помощью пары UV аттосекундных импульсов}

Изложенные выше результаты относились к случаю, когда газ из разреженных атомов водорода возбуждается парой аттосекундных импульсов, распространяющихся на встречу друг другу (рис. 1,a) [25-29]. Однако формула (2) справедлива и для единичного атома (тонкого слоя), возбуждаемого парой импульсов, распространяющихся в одном направлении с некоторой задержкой, рис. $1, b$. Поэтому все изложенные выше результаты рис. 2-5 можно применить и к когерентному возбуждению одиночного атома (тонкого слоя), рис. $1, b$. В этом случае, как отмечалось в $[13,14]$, возможно селективное воздействие пары импульсов на среду. Так, из рис. 2 следует и тривиальный результат - для достижения значительного возбуждения среды необходимо использовать резонансное излучение.

Однако из рис. 4,5 вытекает и менее очевидный результат - возможность эффективного управления, а также селективного возбуждения/девозбуждения населенностей атомных уровней с помощью униполярных субцикловых импульсов по сравнению с биполярными многоцикловыми, что уже отмечалось в $[13,14,29]$. При этом высокая эффективность достигается, несмотря на нерезонансный характер взаимодействия и широкий спектр импульсов.
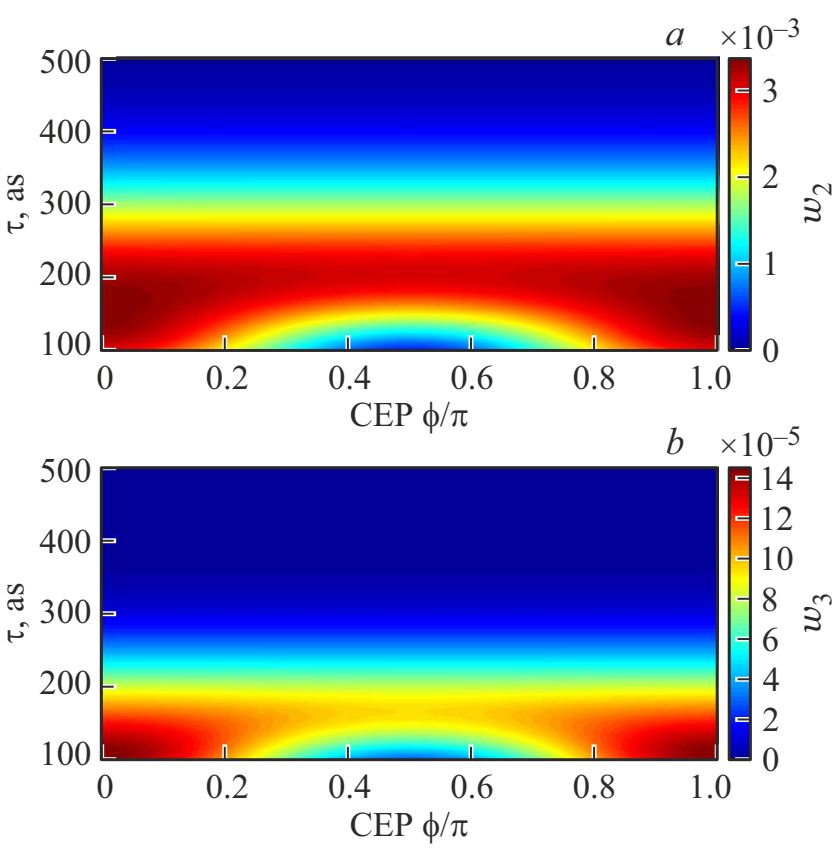

Рис. 5. Зависимость населенностей второго $w_{2}(a)$ и третьего $w_{3}$ уровней $(b)$ в водороде от длительности UV возбуждающих импульсов $\tau$ и СЕР $\phi$ при величине задержки $\Delta=4 \mathrm{fs}$. Остальные параметры указаны в табл. 2.

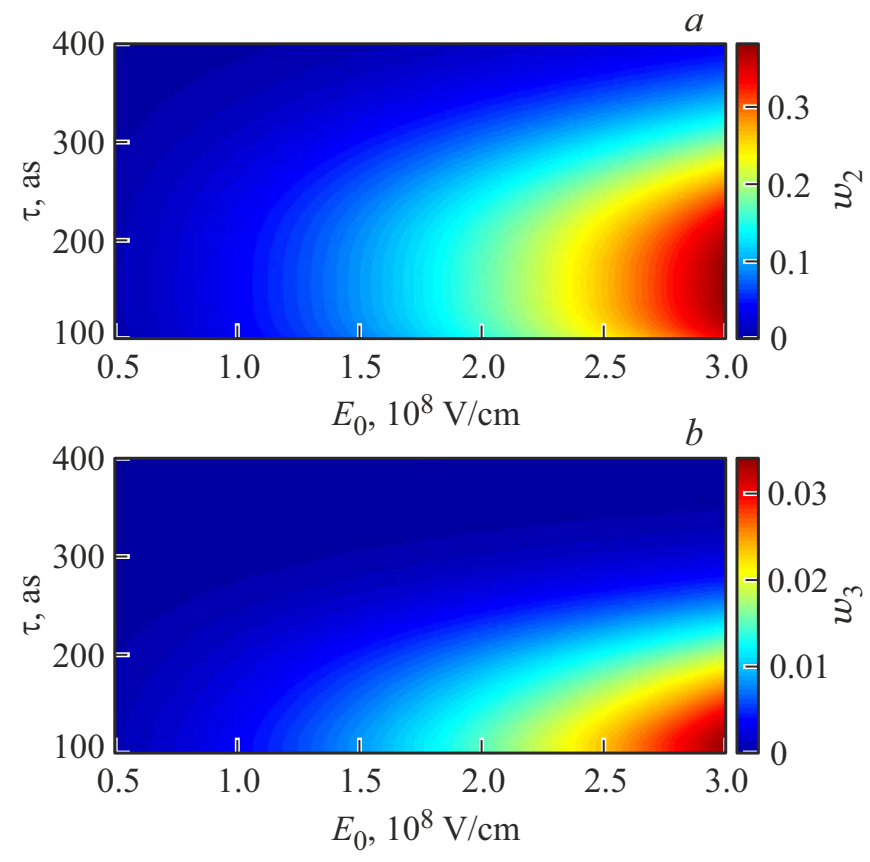

Рис. 6. Зависимость $w_{2}(a)$ и $w_{3}(b)$ в водороде от длительности UV возбуждающих импульсов $\tau$ и амплитуды $E_{0}$ при величине задержки $\Delta=2 \mathrm{fs}$, CEP $\phi=0$.

\section{Заключение}

В работе изучено возбуждение протяженной среды из газообразного водорода (рис. $1, a)$ и одиночного атома водорода (тонкого слоя, рис. $1, b$ ) парой UV аттосекундных импульсов. Показана возможность наведения решеток населенностей в газе из атомарного водорода с помощью пары импульсов, не перекрывающихся в газе при изменении задержки между ними. При резонансном возбуждении для получения решеток с большой глубиной модуляции необходимо использовать импульсы большой длительности. При этом результаты расчета населенностей уровней, полученные по теории возмущений, согласуются с результатами численных расчетов с помощью системы уравнений для матрицы плотности трехуровневой среды.

При нерезонансном возбуждении наиболее эффективное воздействие на одиночный атом и создание решеток в протяженной среде происходит при их возбуждении квазиуниполярными субцикловыми импульсами по сравнению с биполярными многоцикловыми. Этот факт еще раз подтверждает возможность применения униполярного света для эффективного контроля населенностей атомных систем [10,12-14]. Глубина модуляции решеток также сильно зависит от фазы возбуждающих импульсов (CEP) и максимальна в случае использования квазиуниполярных субцикловых импульсов, когда электрическая площадь максимальна.

Исследованные решетки могут найти применения для управления направлением распространения XUV излу- 
чения в пространстве [33], голографической записи [34], измерения времен релаксации поляризации $T_{2}$ [20-23] и др.

\section{Благодарности}

Авторы выражают благодарность И.В. Бабушкину за полезные обсуждения, стимулировавшие авторов к написанию данной заметки.

\section{Финансирование работы}

Исследования динамики населенностей уровней в атоме водорода под действием аттосекундных импульсов выполнены при финансовой поддержке РНФ в рамках научного проекта № 17-19-01097-П. Изучение роли электрической площади во взаимодействии импульсов излучения с микрообъектами поддержано грантом РФФИ 19-02-00312.

\section{Конфликт интересов}

Авторы заявляют, что у них нет конфликта интересов.

\section{Список литературы}

[1] Brabec T., Krausz F. // Rev. Mod. Phys. 2000. V. 72. N 2. P. 545.

[2] Krausz F., Ivanov M. // Rev. Mod. Phys. 2009. V. 81. P. 163.

[3] Calegari F. et al. // J. Phys. B: Atomic, Molecular and Optical Physics. 2016. V. 49. N 6. P. 062001.

[4] Hassan M.T., Luu T.T., Moulet A., Raskazovskaya O., Zhokhov P., Garg M., Karpowicz N., Zheltikov A.M., Pervak V., Krausz F., Goulielmakis E. // Nature. 2016. V. 530. P. 66.

[5] Rossi G.M., Mainz R.E., Yang Y., Scheiba F., SilvaToledo M.A., Chia S.H., Keathley P.D., Fang S., Mücke O.D., Manzoni C., Cerullo G., Cirmi G., Kärtner F.X. // Nature Photonics. 2020. V. 14. N 10. P. 629-635.

[6] Ramasesha K., Leone S.R., Neumark D.M. // Annu. Rev. Phys. Chem. 2016. V. 67. P. 41.

[7] Garg M., Kern K. // Science. 2020. V. 367. N 6476. P. 411-415.

[8] Wu H.-C., Meyer-ter-Vehn J. // Nature Photon. 2012. V. 6. P. 304.

[9] Xu J., Shen B., Zhang X., Shi Y., Ji L., Zhang L., Xu T., Wang W., Zhao X., Xu Z. // Sci. Rep. 2018. V. 8. P. 2669

[10] Архипов Р.М., Архипов М.В., Розанов Н.Н. // Квант. электрон. 2020. T. 50. № 9. C. 801-815; Arkhipov R.M., Arkhipov M.V., Rosanov N.N // Quant. Electron. 2020. V. 50. N 9. P. 801-815.

[11] Архипов Р.М., Архипов М.В., Пахомов А.В., Жукова М.О., Цыпкин А.Н., Розанов Н.Н. // Письма в ЖЭТФ. 2021. T. 113. № 4. C. 237; Arkhipov R.M., Arkhipov M.V., Pakhomov A.V., Zhukova M.O., Tsypkin A.N., Rosanov N.N. // JETP Lett. 2021. V. 113. N 4. P. 242.

[12] Arkhipov R.M., Arkhipov M.V., Babushkin I., Demircan A., Morgner U., Rosanov N.N. // Opt. Lett. 2019. V. 44. N 5. P. 1202.
[13] Архипов Р.М., Архипов М.В., Пахомов А.В., Розанов Н.Н. // Опт. и спектр. 2020. Т. 128. В. 1. C. 106-109; Arkhipov R.M., Arkhipov M.V., Pakhomov A.V., Rosanov N.N // Opt. Spectrosc. 2020. V. 128. N 1. P. 102-105.

[14] Arkhipov R., Pakhomov A., Arkhipov M., Demircan A., Morgner U., Rosanov N., Babushkin I. // Opt. Express. 2020. V. 28. N 11. P. 17020-17034.

[15] Розанов Н.Н., Высотина Н.В. // ЖЭТФ. 2020. Т. 157. № 1. C. 63-66; Rosanov N.N., Vysotina N.V. // JETP. 2020. V. 130. N 1. P. $52-55$.

[16] Aleksandrov I.A., Tumakov D.A., Kudlis A., Shabaev V.M., Rosanov N.N. // Phys. Rev. A. 2020. V. 102. P. 023102.

[17] Розанов Н.Н. // Письма в ЖЭТФ. 2021. Т. 113. № 3. С. 157; Rosanov N.N. // JETP Lett. 2021. V. 113. N 3. P. 145.

[18] Аллен Л., Эберли Дж. Оптический резонанс и двухуровневые атомы. М.: Мир, 1978; Allen L., Eberly J.H. Optical Resonance and Two-level Atoms (NY: Wiley, 1975).

[19] Hughes S. // Phys. Rev. Lett. 1998. V. 81. N 16. P. 3363.

[20] Abella I.D., Kurnit N.A., Hartmann S.R. // Phys. Rev. 1966. V. 141. P. 391.

[21] Штырков Е.И., Лобков В.С., Ярмухаметов Н.Г. // Письма в ЖЭТФ. 1978. Т. 27. № 12. С. 685; Shtyrkov E.I., Lobkov V.S., Yarmukhametov N.G. // JETP Lett. 1978. V. 27. N 12. P. 648.

[22] Штырков Е.И. // Опт. и спектр. 2013. Т. 114. № 1. С. 105; Shtyrkov E.I. // Opt. Spectrosc. 2013. V. 114. N 1. P. 96.

[23] Szczurek M., Kuśnierz M. // Opt. Commun. 1989. V. 74. P. $121-123$.

[24] Eichler H.J., Günter P., Pohl D.W. Laser-Induced Dynamic Gratings. Berlin, Heidelberg, N.Y., Tokyo: Springer-Verlag, 1981.

[25] Arkhipov R.M., Arkhipov M.V., Babushkin I.V., Demircan A., Morgner U., Rosanov N.N. // Opt. Lett. 2016. V. 41. P. 4983.

[26] Arkhipov R.M., Pakhomov A.V., Arkhipov M.V., Babushkin I., Demircan A., Morgner U., Rosanov N.N. // Sci. Rep. 2017. V. 7. N 1. P. 12467.

[27] Архипов Р.М., Архипов М.В., Пахомов А.В., Розанов Н.Н. // Квант. электрон. 2019. Т. 49. С. 958; Arkhipov R.M., Arkhipov M.V., Pakhomov A.V., Rosanov N.N. // Quant. Electron. 2019. V. 49. P. 958.

[28] Архипов P.М. // Опт. и спектр. 2020. Т. 128. В. 11. С. 1732; Arkhipov R.M. // Opt. Spectrosc. 2020. V. 128. N 11. P. 1865.

[29] Arkhipov R.M., Pakhomov A.V., Arkhipov M.V., Babushkin I., Demircan A., Morgner U., Rosanov N.N. // Sci. Rep. 2021. V. 11. Article Number 1961.

[30] Fülop J.A., Tzortzakis S., Kampfrath T. // Advanced Optical Materials. 2020. V. 8. N 3. P. 1900681.

[31] Собельман И.И. Введение в теорию атомных спектров. М.: Наука, 1977.

[32] Розанов Н.Н. Диссипативные оптические солитоны. От микро- к нано- и атто-. М.: Физматлит, 2011.

[33] Drescher L., Kornilov O., Witting T., Reitsma G., Monserud N., Rouzée A., Mikosch J., Vrakking M.J.J., Schütte B. // Nature. 2018. V. 591. P. 91.

[34] Архипов Р.М., Архипов М.В., Розанов Н.Н. // Письма в ЖЭТФ. 2020. Т. 111. В. 9. С. 586; Arkhipov R.M., Arkhipov M.V., Rosanov N.N. // JETP. Lett. 2020. V. 111. N 9. P. 484. 